\title{
Ginkgolide B attenuates collagen-induced rheumatoid arthritis and regulates fibroblast-like synoviocytes-mediated apoptosis and inflammation
}

\author{
Chuanmei Xie ${ }^{1}$, Jing Jiang ${ }^{2}$, Jianping Liu ${ }^{1}$, Guohua Yuan ${ }^{1}$, Zhenyi Zhao ${ }^{1}$ \\ ${ }^{1}$ Department of Rheumatology, the Affiliated Hospital of North Sichuan Medical College, Nanchong, China; ${ }^{2}$ Department of Gynecology and \\ Obstetrics, the Affiliated Hospital of North Sichuan Medical College, Nanchong, China \\ Contributions: (I) Conception and design: C Xie, Z Zhao; (II) Administrative support: J Liu, Z Zhao; (III) Provision of study materials or patients: J \\ Jiang; (IV) Collection and assembly of data: G Yuan; (V) Data analysis and interpretation: C Xie, J Jiang; (VI) Manuscript writing: All authors; (VII) \\ Final approval of manuscript: All authors. \\ Correspondence to: Guohua Yuan. Department of Rheumatology, the Affiliated Hospital of North Sichuan Medical College, 63 Wenhua Road, \\ Shunqing District, Nanchong, China. Email: vg5gzj0neqn@sina.com.
}

Background: Rheumatoid arthritis (RA) is a systemic disease characterized by chronic synovial infiltration and proliferation, cartilage destruction, and joint injury. Ginkgolide B (GB) is an extract of the leaves of Ginkgo biloba, and pharmacological studies have shown that it has anti-inflammatory and anti-apoptotic activities. The purpose of this study was to investigate the anti-RA properties of GB.

Methods: In vivo, we established a collagen II-induced arthritis (CIA) mouse model. Mice were divided into five groups $(\mathrm{n}=10)$ : sham, CIA, GB $(10 \mu \mathrm{M})$, GB $(20 \mu \mathrm{M})$, and GB $(40 \mu \mathrm{M})$. We measured arthritis score, synovial histopathological change, and peripheral blood cytokine levels. In vitro, we used lipopolysaccharide (LPS)-induced-fibroblast-like synoviocytes (RA-FLSs) as the study subject. Cell viability, apoptosis, and inflammatory cytokines levels were detected by 3-(4,5)-dimethylthiahiazo (-z-y1)-3,5-diphenytetrazoliumromide (MTT) assay, flow cytometry, and quantitative real-time reverse transcription polymerase chain reaction (qRT-PCR), respectively. Finally, the protein expression of wingless-type family member 5A (Wnt5a), c-Jun N-terminal kinase (JNK), and nuclear factor kappa-light-chain-enhancer of activated B cells (NF- $\kappa$ B) p65 were detected by Western blot.

Results: Arthritis scores, synovial hyperplasia, and cartilage and bone destruction were significantly ameliorated by GB. Additionally, GB decreased the serum levels of interleukin (IL)-1 $\beta$, IL-6, monocyte chemoattractant protein-1 (MCP-1), and tumor necrosis factor alpha (TNF- $\alpha$ ), matrix metalloproteinase (MMP)-3 and MMP-13, and increased IL-10. In vitro, we found that GB remarkably inhibited RA-FLSs viability at 24 or $48 \mathrm{~h}$ in a concentration-dependent manner. The apoptotic ratio was reduced by GB, and it increased the expression of cleaved-Caspase- 3 and Bax while decreasing Bcl-2 expression in RA-FLSs. Furthermore, GB attenuated the progression of inflammation by mediating inflammatory cytokine release and MMPs gene expression. Meanwhile, GB inactivated the expression levels of Wnt5a, phosphorylated (p)JNK, and p-P65 in the synovial tissues and RA-FLSs.

Conclusions: This study was the first to demonstrate that the anti-RA effect of GB is related to reducing articular cartilage and bone destruction, inducing RA-FLSs apoptosis, and regulating inflammatory cytokine release and the Wnt5a/JNK/NF- $\mathrm{BB}$ axis. All the findings highlight that GB might provide a novel treatment approach for RA.

Keywords: Ginkgolide B (GB); rheumatoid arthritis (RA); fibroblast-like synoviocytes (FLSs); inflammation

Submitted Aug 28, 2020. Accepted for publication Nov 09, 2020.

doi: $10.21037 / \mathrm{atm}-20-6420$

View this article at: http://dx.doi.org/10.21037/atm-20-6420

^ ORCID: 0000-0001-6114-7426 


\section{Introduction}

Rheumatoid arthritis (RA) is a common chronic autoimmune disease. As a serious extra-articular disease, RA is characterized by synovial hyperplasia, and joint and cartilage damage, which leads to joint pain, swelling, and other complications in patients $(1,2)$. The incidence rate of RA in the global population is about $0.5 \%$ (3), and although RA is not a direct cause of death, it greatly reduces patients' quality of life and ability to work (4). Synovial inflammation is the main pathological change of RA; activated fibroblastlike synoviocytes (FLSs) show tumor-like features of excessive proliferation, anti-apoptosis, migration, and invasion (5). The FLSs also play a vital role in the pathogenesis of RA through releasing proinflammatory cytokines such as TNF- $\alpha$, interleukin-(IL)-1 and IL-6 (6). Unfortunately, RA is difficult to diagnose and treat in the early stage, and current treatment involves regular health checks and long-term control medication. Therefore, it is crucial to find new therapeutic strategies for the treatment of RA.

Ginkgo biloba (Ginkgoaceae) is a woody plant, the extracts of which are widely used across China and Europe (7) because of the protective effect it offers for various cardiovascular and nerve diseases $(8,9)$. Ginkgolide B (GB, a diterpene lactone compound) is extracted from Ginkgo biloba leaves, and has been shown to have antiinflammatory, anti-apoptotic, and anti-oxidative effects. For instance, GB improves nerve function by promoting the proliferation and differentiation of rat neural stem cells (10). In addition, GB inhibits the platelet-activating factorinduced inflammatory cascade effect (11). Furthermore, GB reduces osteoporosis by promoting osteoblast differentiation (12), and plays an anti-inflammatory role in lipopolysaccharide (LPS)-induced chondrocytes (13). However, the protective role of GB in the case of RA has not yet received much attention.

Wingless-type (Wnt) proteins regulate cell proliferation, differentiation, and survival, and play an important role in embryonic development (14). The development of cartilage, bone, and articular cavities are highly dependent on Wnt signaling (15). The Wnt isoform $W n t 5 a$, is associated with some inflammatory diseases. Recent reports have indicated that $W n t 5 a$ was involved in IL-1 $\beta$-induced cell migration and differentiation (16), and Wnt5a silencing inhibited IL-1 $\beta$-induced degradation of type II collagen in rat chondrocytes (17). Furthermore, some researchers have asserted that $W n t 5 a$ is an inflammatory factor that mediates the release of proinflammatory cytokines, such as IL-6, TNF- $\alpha$, and interferon, among others (18). Therefore, here we hypothesized that $W n t 5 a / \mathrm{JNK} / \mathrm{NF}-\kappa \mathrm{B}$ signaling cascades might be involved in RA inflammation.

In this study, our aim was to study the protective role of GB in the collagen II-induced RA mouse model, evaluate the effect on RA-FLSs apoptosis, proliferation, and inflammatory response, and attempt to find the mechanism of its pharmacological activity.

We present the following article in accordance with the ARRIVE reporting checklist (available at http://dx.doi. org/10.21037/atm-20-6420).

\section{Methods}

\section{Main materials}

GB (formula: $\mathrm{C}_{20} \mathrm{H}_{24} \mathrm{O}_{10}$; M.wt: 424.4; purity: >98\%) was obtained from R\&D Systems (Minneapolis, MN, USA). Antibodies were obtained from Abcam (Cambridge, UK) or Cell Signaling Technology (CST, Danvers, MA, USA). Complete Freund's adjuvant (CFA) and immunization grade bovine collagen II (CII) were obtained from Sigma-Aldrich (St. Louis, MO, USA). si-Wnt5a (sense GGUCCCUAGGUAUGAAUAATT, an-tisense UUAUUCAUACCUAGGGACCTT) was obtained from Gima gene (Wuhan, China). Five $\mu$ M SP600125 was obtained from Sigma-Aldrich (St. Louis, MO, USA).

\section{Collagen-induced arthritis (CIA) model and animals administration}

Male DBA/1J mice (8 weeks old) were obtained from the animal center of North Sichuan Medical College (Nanchong, Sichuan, China). Animal protocols in this study were approved by the Animal Care and Use Committee of North Sichuan Medical College. All animal experiments were performed in accordance with the guidelines of the Animal Care and Use Committee of North Sichuan Medical College. The method of creating the CIA model was as previously reported (19). On days 1 and 7, $100 \mu \mathrm{g}$ of bovine CII were dissolved in $0.1 \mathrm{mM}$ acetic acid and emulsified with CFA $(1: 1, \mathrm{w} / \mathrm{v})$ at the base of tail; the same amount of saline was administered for the normal control group mice. Post primary immunization, on day 25, an intraperitoneal booster vaccine was injected with the same preparation of $\mathrm{CII}$ in Freund's incomplete adjuvant. Twice a week, the mice body weights were measured and clinical scores assessed. All 
experimental mice were fed orally from day 26 to 43 , and were randomly divided into 5 groups $(\mathrm{n}=10)$ : sham, CIA, GB $(10 \mu M)$, GB $(20 \mu M)$, and GB $(40 \mu M)$.

\section{Clinical evaluation}

Mice weight and arthritis levels were measured every 3 days as above. Arthritis scores were determined as previously described, with a maximum score of 16 per mouse (20). The quantitative and systematic classification of arthritis severity was as follows: 0 -normal joint; 1 -slight swelling or onedigit erythema; 2-skin redness, slight swelling of ankle and foot; 3-moderate swelling and erythema; 4-severe swelling and erythema (paw or fore paw).

\section{Histological analysis}

After 35 days, the animals were sacrificed and soaked in $75 \%$ alcohol. Knee joints were fixed in $10 \%$ formaldehyde for 1 week. A month after decalcification, the tissues were embedded in paraffin and sliced at $5 \mu \mathrm{m}$ thickness. The sections were then stained with hematoxylin and eosin (H\&E) and Alcian blue staining $(21,22)$ to analyze synovial inflammation and cartilage destruction. The stained sections were sealed with a neutral adhesive and observed under a microscope (Olympus CX23, Tokyo, Japan).

\section{Serum cytokines detection}

Mice were sacrificed and blood was collected from the tail vein. Blood samples were centrifuged at 3,000 rpm for $10 \mathrm{~min}$, then serum was extracted and stored $-80^{\circ} \mathrm{C}$. Serum cytokines [IL-1 $\beta$, IL-6, monocyte chemoattractant protein-1 (MCP-1), IL-10, TNF- $\alpha$, matrix metalloproteinase 3 (MMP-3), and MMP-13] were analyzed by an enzyme linked immunosorbent assay (ELISA) kit (R\&D Systems, Minneapolis, MN, USA) according to the manufacturer's instructions.

\section{Cell culture}

The primary FLSs were obtained from synovial tissues (23). As previously described, dissociated cells were cultured in Dulbecco's Modified Eagle Medium (DMEM) medium (Thermo Fisher, Waltham, MA, USA) containing 10\% heat-inactivated fetal bovine serum (FBS) (Gibco, Grand Island, NY, USA), $100 \mathrm{U} / \mathrm{mL}$ penicillin, and $100 \mu \mathrm{g} / \mathrm{mL}$ streptomycin (Sigma-Aldrich, St Louis, MO, USA), and placed in a $37{ }^{\circ} \mathrm{C}, 5 \% \mathrm{CO}_{2}$ constant incubator. Normal FLSs were stimulated with $1 \mu \mathrm{g} / \mathrm{mL}$ LPS (24).

\section{Cell toxicity and viability}

The RA-FLSs were seeded into 96-well plates at a density of $1 \times 10^{5}$ cells/well, and the cell viability was determined by 3-(4,5)-dimethylthiahiazo (-z-y1)-3,5-diphenytetrazoliumromide (MTT) assay after incubation with a gradient of GB concentrations $(0,5,10,20,40$, and $80 \mu \mathrm{M}$ ) for 24 or $48 \mathrm{~h}$. About $20 \mu \mathrm{L}$ MTT solution (Abcam, Cambridge, UK) was added to each well and then rested at $37^{\circ} \mathrm{C}$ for $3 \mathrm{~h}$. After removing the supernatant, $100 \mu \mathrm{L}$ dimethyl sulfoxide (DMSO) was added to each well and oscillated at low speed for $15 \mathrm{~min}$ to fully dissolve the crystal. The absorbance at $490 \mathrm{~nm}$ was analyzed using a RNE90002 microplate reader (Reagen LLC, Moorestown, NJ, USA).

\section{Flow cytometry}

Apoptosis of RA-FLSs was assessed using an Annexin V-FITC/PI cell apoptosis detection kit (CST, Boston, MA, USA). Simply put, RA-FLSs were exposed to 10 or $20 \mu \mathrm{M}$ GB for $48 \mathrm{~h}$. Then, $5 \times 10^{5} \mathrm{RA}-\mathrm{FLSs}$ were digested by trypsin and centrifuged at $1,000 \mathrm{rpm}, 4^{\circ} \mathrm{C}$ for $5 \mathrm{~min}$. After washing with phosphate buffer saline (PBS) twice, RAFLSs were re-suspended in $100 \mu \mathrm{L}$ of binding buffer, and incubated with $10 \mu \mathrm{L}$ fluorescein isothiocyanate (FITC) and $5 \mu \mathrm{L}$ propidium iodide (PI) solution at room temperature (RT) in the dark for $15 \mathrm{~min}$. Finally, apoptotic cells were analyzed by an Attune NxT flow cytometry (Thermo Fisher, Waltham, MA, USA) within $1 \mathrm{~h}$.

\section{Quantitative real-time polymerase chain reaction ( $q R T$ - PCR)}

Total RNA was extract from RA-FLSs using TRIzol reagents (Invitrogen, Carlsbad, CA, USA), and the purity of total RNA was measured using a Nanodrop ${ }^{\text {TM }}$ 1000 spectrophotometer (Thermo Fisher, Waltham, MA, USA). Then, RNA was reverse transcribed into cDNA by a PrimeScript ${ }^{\mathrm{TM}} \mathrm{RT}$ reagent kit (TaKaRa, Otsu, Shiga, Japan), target genes were amplified using $\mathrm{SYBR}^{\circledR}$ Premix

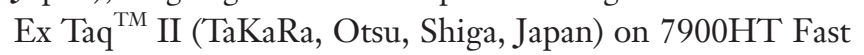
Real-Time PCR System (Thermo Fisher, Waltham, MA, USA). Relative mRNA levels were calculated using the $2^{-\Delta \Delta \mathrm{Ct}}$ method, repeated 3 times. 

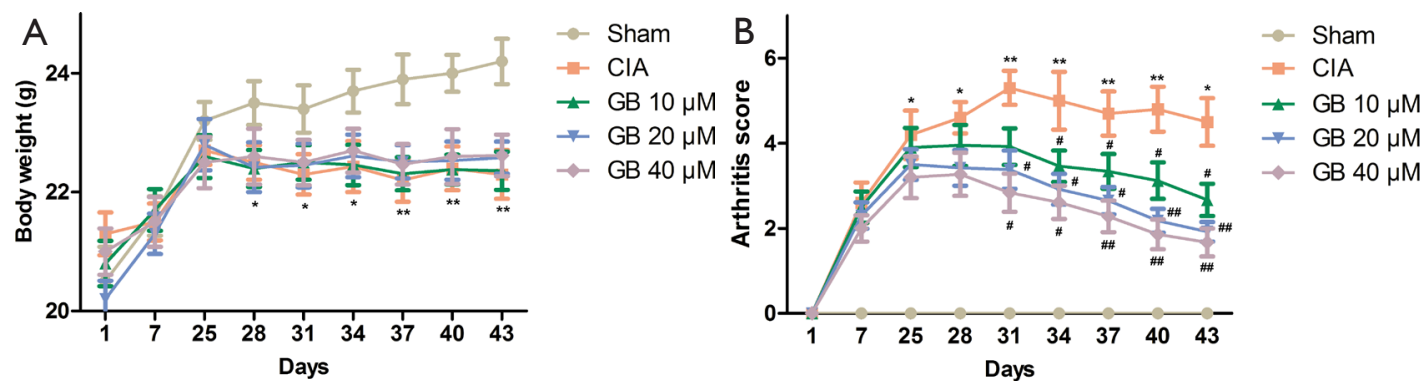

Figure 1 Effect of GB on the arthritis severity in RA mice. Mice were randomly divided into five groups after the booster immunization on day 25 and were orally administered GB from day 25 to the day 43. (A) Effect of GB on body weight; (B) effect of GB on the arthritis score. Data were presented as mean \pm standard deviation $(\mathrm{SD}), \mathrm{n}=10 .{ }^{*}, \mathrm{P}<0.05,{ }^{* *}, \mathrm{P}<0.01$, vs. sham group; ${ }^{\#}, \mathrm{P}<0.05,{ }^{\# \#}, \mathrm{P}<0.01$, s. $\mathrm{CIA}$ group. GB, Ginkgolide B; RA, rheumatoid arthritis; CIA, collagen II-induced arthritis.

\section{Western blot}

Synovial tissues and RA-FLSs were used for protein extraction. After being denatured, the protein samples were separated by $10 \%$ sodium dodecyl sulfate-polyacrylamide gel (SDS-PAGE) and transferred to polyvinylidene fluoride (PVDF) membranes. Then, the protein samples were placed in RT 5\% skim milk and sealed for $2 \mathrm{~h}$; the membranes were incubated with primary antibodies at $4{ }^{\circ} \mathrm{C}$ overnight, after which they were incubated with goat anti-rabbit IgG (ab6721, Abcam, Cambridge, UK) at RT for 1h. Following that, the bands were visualized by enhanced chemiluminescent (ECL) kit (Bio-Rad, Berkeley CA, USA). Antibodies were listed below: anti-wnt5a (ab229200, Abcam), Anti-JNK antibody (\#9252, CST), Anti-p-JNK A antibody (\#9255, CST), Anti-P65 antibody (ab16502, Abcam), Anti-p-P65 (ab86299, Abcam), and $\beta$-actin (ab8226, Abcam).

\section{Statistical analysis}

All experimental operations were repeated at least 3 times. Data was analyzed using SPSS 22.0 software (IBM, Chicago, IL, USA) and GraphPad Prism 5.0 (GraphPad software, La Jolla, CA, USA), and presented as mean \pm standard deviation (SD). The comparison between multiple groups was done using one-way ANOVA following by post hoc testing. A significant difference was considered to be $\mathrm{P}<0.05$.

\section{Results}

The body weight and arthritis score of CIA mice was mediated by $G B$

During the experiment, the weight of mice in the sham group steadily increased. Compared with the sham group, CIA mice lost weight after the second immunization and then it slowly increased. Moreover, GB treatment (10, 20, or $40 \mu \mathrm{M})$ had no significant effect on mouse body weight, compared with the CIA group (Figure 1A). In addition, the average arthritis score demonstrated that the CIA model had been successfully established (Figure 1B). On day 31, the symptoms of arthritis in the CIA group reached a peak, which was significantly different from the sham group. Compared with the CIA group, GB treatment significantly reduced arthritis symptoms from day 38. During the whole experimental period, we monitored the physical state and behavior of mice, and found that GB treatment had no obvious adverse effect on mice.

\section{Collagen II-induced joint injury was alleviated by GB in $R A$ mice}

In order to further study the effect of GB on CIA mice, histological evaluation of mouse ankle joints was conducted by $\mathrm{H} \& \mathrm{E}$ and alcian blue staining. As shown in Figure $2 A, B$, there was complete joint structure, smooth cartilage surface, and no infiltration in the sham group. Nevertheless, in CIA mice, we observed significant synovial hyperplasia, pannus formation, inflammatory cell infiltration, and cartilage degeneration. Treatment with $\mathrm{GB}(10,20$, or $40 \mu \mathrm{M})$ improved these pathological changes and ameliorated the severity of cartilage damage, especially in the $40 \mu M$ group. The histological score of the CIA + GB group treatment was significantly lower than that of the CIA group (Figure 2C), and the area of chondrocyte loss in the GB administration groups was reduced in a dose-dependent manner (Figure 2D). 
A

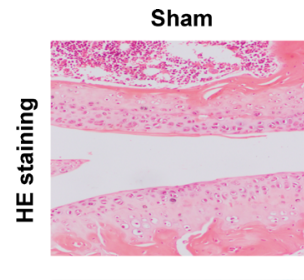

CIA

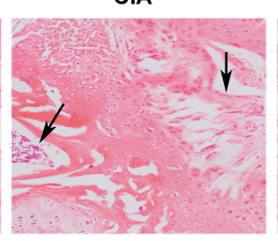

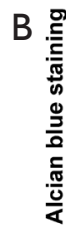

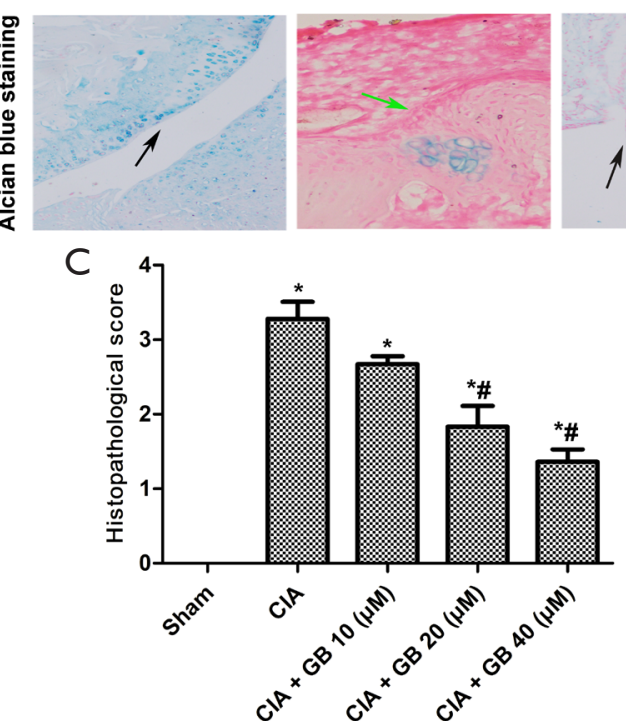

GB $10 \mu M$
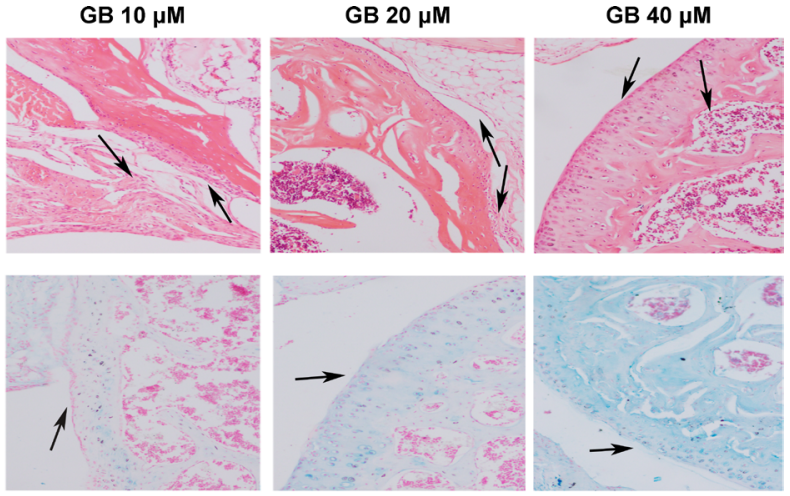

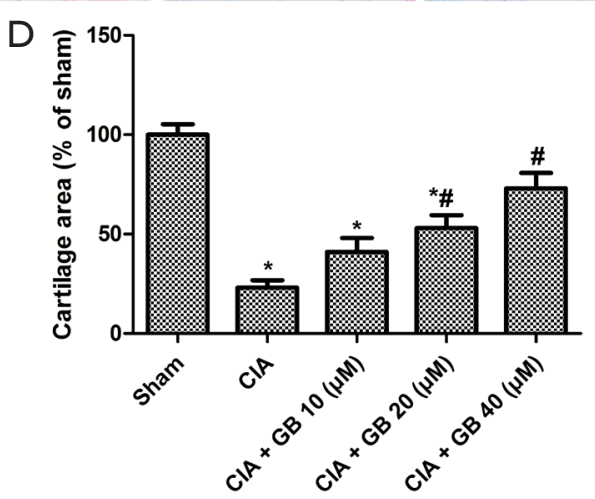

Figure 2 Effect of GB on joint injury in RA mice. (A) Ankle joints tissues were stained using H\&E stain, representative images were magnified at $\times 100$. Black arrow, infiltration of inflammatory cells or hyperplasia of synovia; (B) representative Alcian blue staining, images were magnified at $\times 100$. Black arrow, the clear articular cartilage layer. Green arrow, serious loss of chondrocytes; (C) histology score of each group; (D) cartilage area of each group. Data were presented as mean \pm standard deviation (SD), $\mathrm{n}=10$. * $\mathrm{P}<0.05$, vs. sham group; ${ }^{*}, \mathrm{P}<0.05$, vs. CIA group. GB, Ginkgolide B; RA, rheumatoid arthritis; H\&E, hematoxylin and eosin.

\section{GB attenuated collagen II-induced inflammatory response and MMPs content in RA mice}

Inflammatory cytokines play an important role in synovial hyperplasia and bone damage in RA, this study explored the effect of $\mathrm{GB}$ on the level of inflammatory factors in mice models. As shown in Figure $3 A, B, C, D, E$, compared with the sham group, the serum levels of pro-inflammatory cytokines (IL-1 $\beta$, IL-6, MCP-1, and TNF- $\alpha$ ) were markedly higher and anti-inflammatory cytokine (IL-10) was obviously lower in CIA mice. Importantly, GB treatment decreased the levels of pro-inflammatory cytokines and increased the levels of anti-inflammatory cytokines. Next, we analyzed the serum production of MMP-3 and MMP-13 in CIA mice. Figure $3 F, G$ showed that the serum levels of MMP3 and MMP-13 in the CIA mice were markedly elevated, compared with the sham group. Treatment with GB (10, 20 , or $40 \mu \mathrm{M}$ ) significantly decreased MMPs (MMP-3 and MMP-13) expression, compared with those observed in the
CIA group.

\section{Treatment with GB inbibited proliferation and induced apoptosis of RA-FLSS}

To investigate the effects of GB on RA-FLSs viability, RAFLSs were treated with different concentrations of GB $(0,5,10,20,40$, or $80 \mu \mathrm{M})$ for 24 and $48 \mathrm{~h}$, respectively. As shown in Figure 4A, MTT analysis showed that there was no cytotoxic effect when the GB concentration was $<80 \mu \mathrm{M}$ at $24 \mathrm{~h}$. When the concentration was $>40 \mu \mathrm{M}$, GB had cytotoxicity on the RA-FLSs at $48 \mathrm{~h}$. Therefore, we understood $40 \mu \mathrm{M}$ to be the highest concentration in the safe range, and the GB concentration in the following experiments was 10 and $20 \mu \mathrm{M}$. In addition, apoptosis was detected using flow cytometry, and we found that GB obviously elevated the ratio of apoptotic cells, compared with the control (Figure 4B,C). Furthermore, Western blot confirmed that GB increased the expression of Caspase-3 

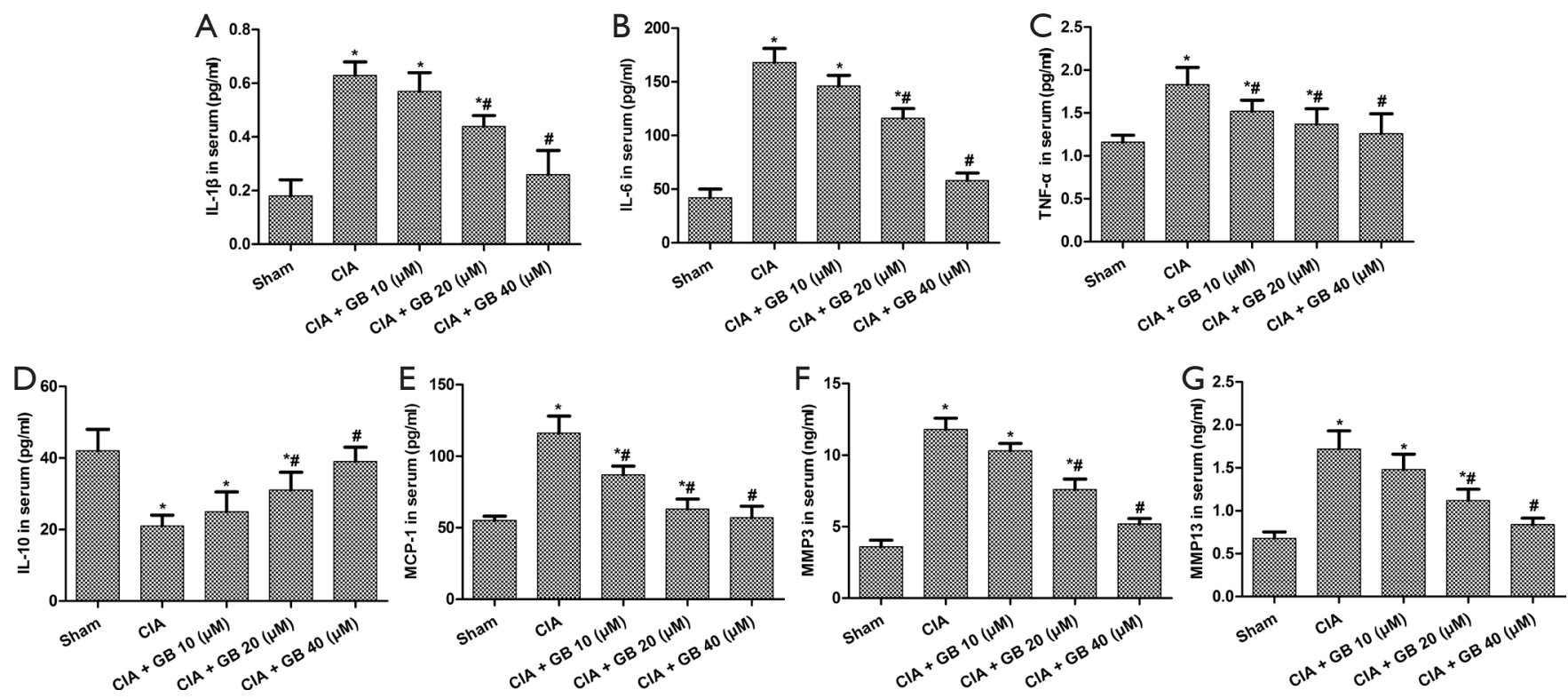

Figure 3 Effect of GB on the inflammatory response in RA mice. (A,B,C,D,E,F,G) The serum level of IL-1 $\beta$, IL-6, MCP-1, TNF- $\alpha$, IL-10, MMP-3, and MMP-13 were measured by ELISA. Data were presented as mean \pm standard deviation (SD), $\mathrm{n}=10 .{ }^{*}, \mathrm{P}<0.05, v s$. sham group; , $\mathrm{P}<0.05$, vs. CIA group. MMP, matrix metalloproteinase; ELISA, enzyme-linked immunosorbent assay; CIA, collagen II-induced arthritis.

and Bax, while it decreased Bcl-2 expression in RA-FLSs (Figure 4D).

\section{Inflammatory cytokine secretion and cartilage-gene expression in RA-FLSs was decreased by $G B$}

In vivo studies showed that GB could regulate inflammation. Therefore, we further detected the mRNA expression of IL-1 $\beta$, IL-6, MCP-1, TNF- $\alpha$, and IL-10 in RA-FLSs by qRT-PCR. Data indicated that in the CIA group, the levels of IL-1 $\beta$, IL-6, MCP-1, and TNF- $\alpha$ were significantly increased, and IL-10 was decreased. However, the expression of these cytokines were reversed by GB 10 or $20 \mu \mathrm{M}$ treatment (Figure $5 A, B, C, D, E$ ). Pretreatment with GB significantly decreased and matrix-degrading genes (MMP-3, MMP-13) in RA-FLSs in protein level (Figure 5F).

\section{The proteins Wnt $5 a, 7 N K$ and $N F-\kappa B$ were inactivated by $G B$}

In order to study underlying signaling pathways in vivo and in vitro, we examined the relative protein expression of $W n t 5 a$, JNK and NF-kB in synovial tissue and RA-FLSs. As shown in Figure 6A, the expression levels of $W n t 5 a$, p-JNK, and p-P65 were significantly elevated in the synovial tissues of mice in the CIA group, compared with sham group. By contrast, GB treatment $(10,20$, or $40 \mu \mathrm{M})$ markedly decreased their protein levels. In parallel, we found that the expression trend of these proteins in RA-FLSs was consistent with that in the synovium, and 10 or $20 \mu \mathrm{M}$ GB obviously reduced the expression of $W n t 5 a, \mathrm{p}-\mathrm{JNK}$, and p-P65, compared with control group (Figure 6B).

\section{GB attenuates rbeumatoid arthritis through inbibiting the Wnt $5 a / f N K / N F-\kappa B$ patbway}

Furthermore, RA-FLSs cells were treated with Wnt5a siRNA. We found that the expression of $\mathrm{p}-\mathrm{JNK} / \mathrm{JNK}$ and p-P65 in si-Wnt5a group were lower than those in GB $(20 \mu M)$ group. There was no significant difference of Wnt5a expression in si-Wnt5a group or GB group. The expression of Wnt5a, p-JNK/JNK, and p-P65 were further reduced in si-Wnt5a + GB group. These results considered that combination of GB and si-Wnt5a has synergistic inhibitory effect on activated Wnt5a/JNK/NF- $\mathrm{kB}$ signaling pathway in RA-FLSs cells. Then, JNK inhibitor (SP60025) was used to further study the underlying mechanisms of GB inhibition of Wnt5a/JNK/NF- $\kappa$ B. Interestingly, the result in SP60025 + GB group was similar to those of siWnt5a + GB group, and the p-P65 expression was higher in the SP60025 group than that in the si-Wnt5a group or si- 

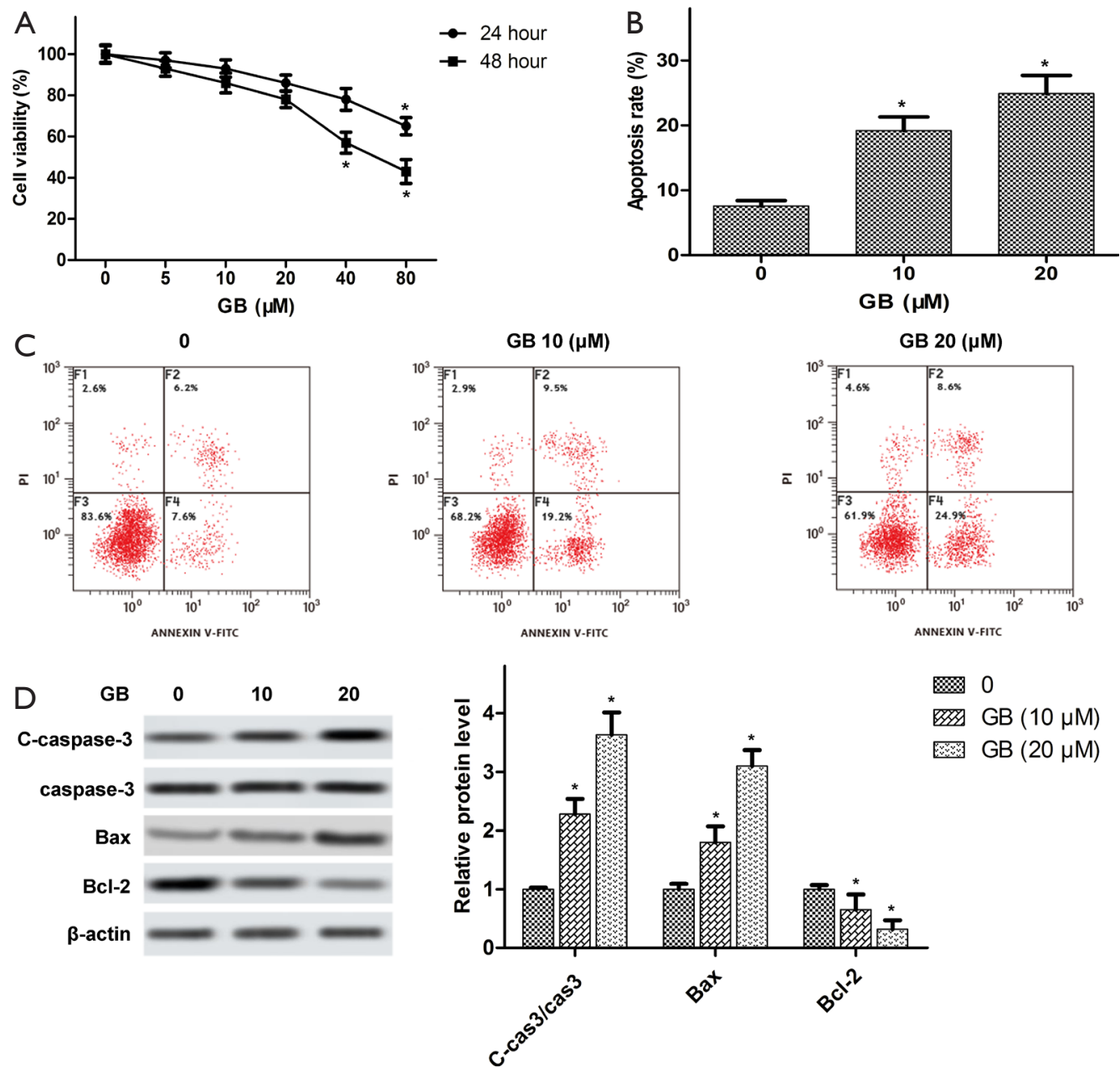

Figure 4 Effect of GB on RA-FLSs proliferation and apoptosis. (A) RA-FLSs were treated with pectolinarin (0, 5, 10, 20, or 30 $\mu$ M) for 24 or $48 \mathrm{~h}$, then cell viability was evaluated by MTT; (B,C) the apoptosis ratio of RA-FLSs was tested by flow cytometry; (D) the protein levels of cleaved-Caspase-3, Bax and Bcl-2 in RA-FLSs were detected by Western blot. Data were presented as mean \pm standard deviation (SD). *, $\mathrm{P}<0.05$, vs. control group. GB, Ginkgolide B; RA-FLS, rheumatoid arthritis fibroblast-like synoviocytes; CIA, collagen II-induced arthritis.

Wnt5a + GB group (Figure $7 A, B, C)$.

\section{Discussion}

A widespread chronic arthropathy, RA is accompanied by synovial hyperplasia and aggressive inflammation. The CIA animal model is widely used in RA therapy (19), and RA-FLSs is considered to be an important effector cell to maintain synovial homeostasis (25). The current study examined the inflammatory cytokines and signaling pathways of RA pathogenesis. Our aim was to explore the therapeutic effects of GB on type II collagen arthritis in mice, and its effects on RA-FLSs proliferation, apoptosis, and inflammation. Here, we demonstrated for the first time that GB through inactivation of the $W n t 5 a / \mathrm{JNK} / \mathrm{NF}-\kappa \mathrm{B}$ pathway attenuated rheumatoid joint injury, inhibited RAFLSs proliferation, promoted apoptosis, and regulated the release of inflammatory cytokines.

In RA patients, the dysregulation of cytokines induces inflammatory responses, stimulates osteoclast differentiation, and engenders synovial hyperplasia and progressive joint destruction $(26,27)$. As far as we know, GB is a widely used anti-inflammatory plant. In this study, we established in vivo CIA mouse models and in vitro LPSinduced FLSs, to elucidate the anti-arthritic role of GB. The findings showed GB improved the paw swelling and 

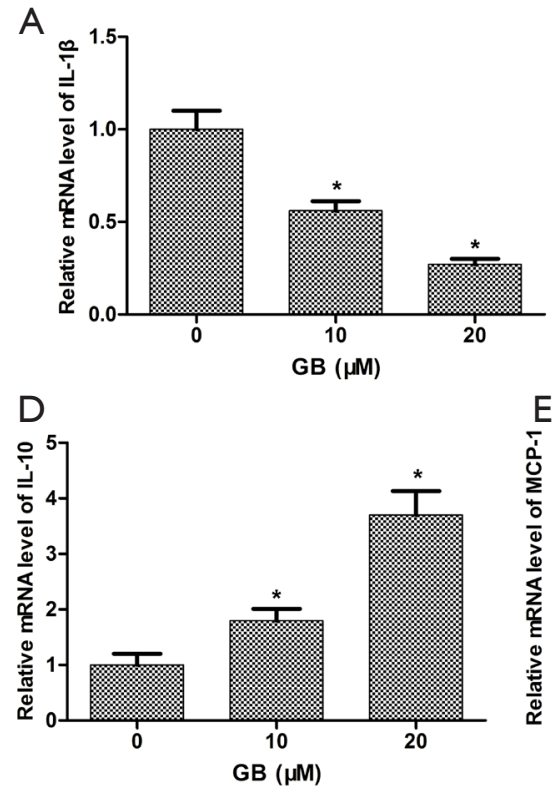
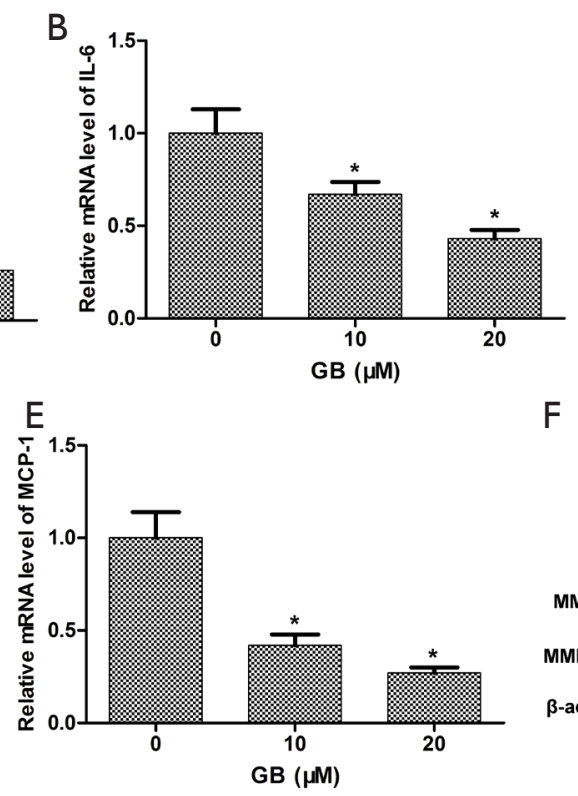

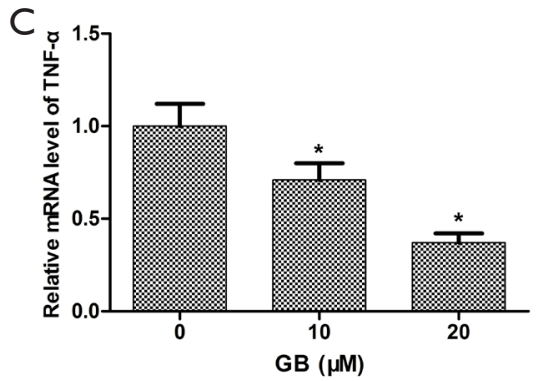

F
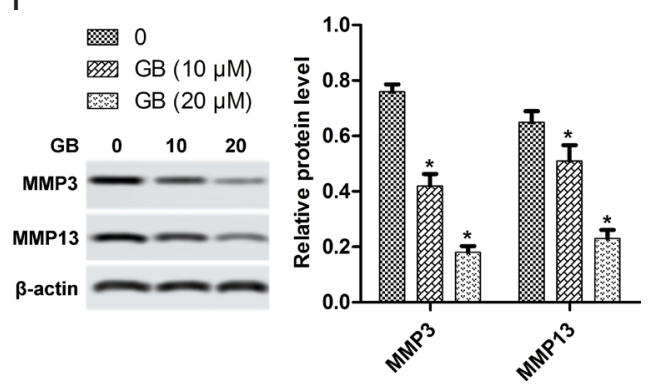

Figure 5 Effect of GB on the inflammatory cytokine secretion and MMPs expression in RA-FLSs. (A,B,C,D,E) The mRNA levels of IL$1 \beta$, IL-6, MCP-1, TNF- $\alpha$, and IL-10 in RA-FLSs were examined by qRT-PCR; (F) the protein levels of MMP-3 and MMP-13 in RAFLSs were detected by Western blot. Data were presented as mean \pm standard deviation (SD). * $\mathrm{P}<0.05$, vs. control group. MMPs, matrix metalloproteinases; RA-FLS, rheumatoid arthritis fibroblast-like synoviocytes; qRT-PCR, quantitative real-time polymerase chain reaction; CIA, collagen II-induced arthritis.

arthritic lesions of CIA mice, and alleviated cartilage and bone damage in vitro. Generally, GB is considered to be the most specific natural platelet activating factor (PAF) antagonist with high biological activity, and has been shown to be involved in cell survival and death (28). Our conclusions supported the hypothesized role that GB effectively stimulated RA-FLSs apoptosis and suppressed the levels of IL-1 $\beta$, IL-6, MCP-1, and TNF- $\alpha$ in synovia and sera.

The onset of RA leads to the release of pro-inflammatory cytokines, such as TNF- $\alpha$, IL- 6 , and IL-1 $\beta$ (29). On the other hand, inflammatory cytokines [TNF- $\alpha$, IL-6, IL- $1 \beta$, or granulocyte-macrophage colony-stimulating factor (GMCSF)] induce the synovitis inflammatory cascade, including synovial hypertrophy, and cartilage and bone invasion (30). Additionally, IL-1 $\beta$ mediates synoviocyte-induced cell proliferation and cartilage degradation (31). Activated FLSs secrete mediators such as TNF- $\alpha$, IL- $1 \beta$, and chemokines, and penetrate into the synovium to indirectly stimulate bone erosion and irreversible joint injury (32). This study showed that treatment with GB suppressed the levels of these pro-inflammatory cytokines in the serum of CIA mice and RA-FLSs. Furthermore, pro-inflammatory cytokines (such as TNF- $\alpha$, IL-1 $\beta$, IL-6, and IL-8) also promoted the secretion of MMPs $(33,34)$. MMPs belongs to the family of zinc/calcium-dependent endopeptidase and is produced by synovial fibroblasts. Elevated MMP levels exacerbate collagen fiber digestion and degradation of extracellular matrix, leading to joint and synovial tissue damage $(33,35)$. In our study, we found that treatment with GB decreased the expression of MMP-3 and MMP-13 both in the serum of CIA mice and RA-FLSs. Therefore, combined with the pathological results of synovial tissue and in vitro experiments, it is suggested that GB has a strong protective effect on rheumatoid arthritis. Therefore, in combination with the results of histopathological examinations of joints and synovial tissues, suggesting that GB exerted a strong protective effect in rheumatoid arthritis, which could be used as a candidate drug for the treatment of rheumatoid arthritis.

We focused on the NF- $\kappa \mathrm{B}$ and JNK pathways in our study due to their important role in cellular inflammation and cartilage degradation. Studies have shown that NF-кB/ p65 plays a key role in the transcription of inflammatory cytokines and chemokines (36). The NF- $\mathrm{KB}$ signaling pathway is highly activated in the collagen-induced 

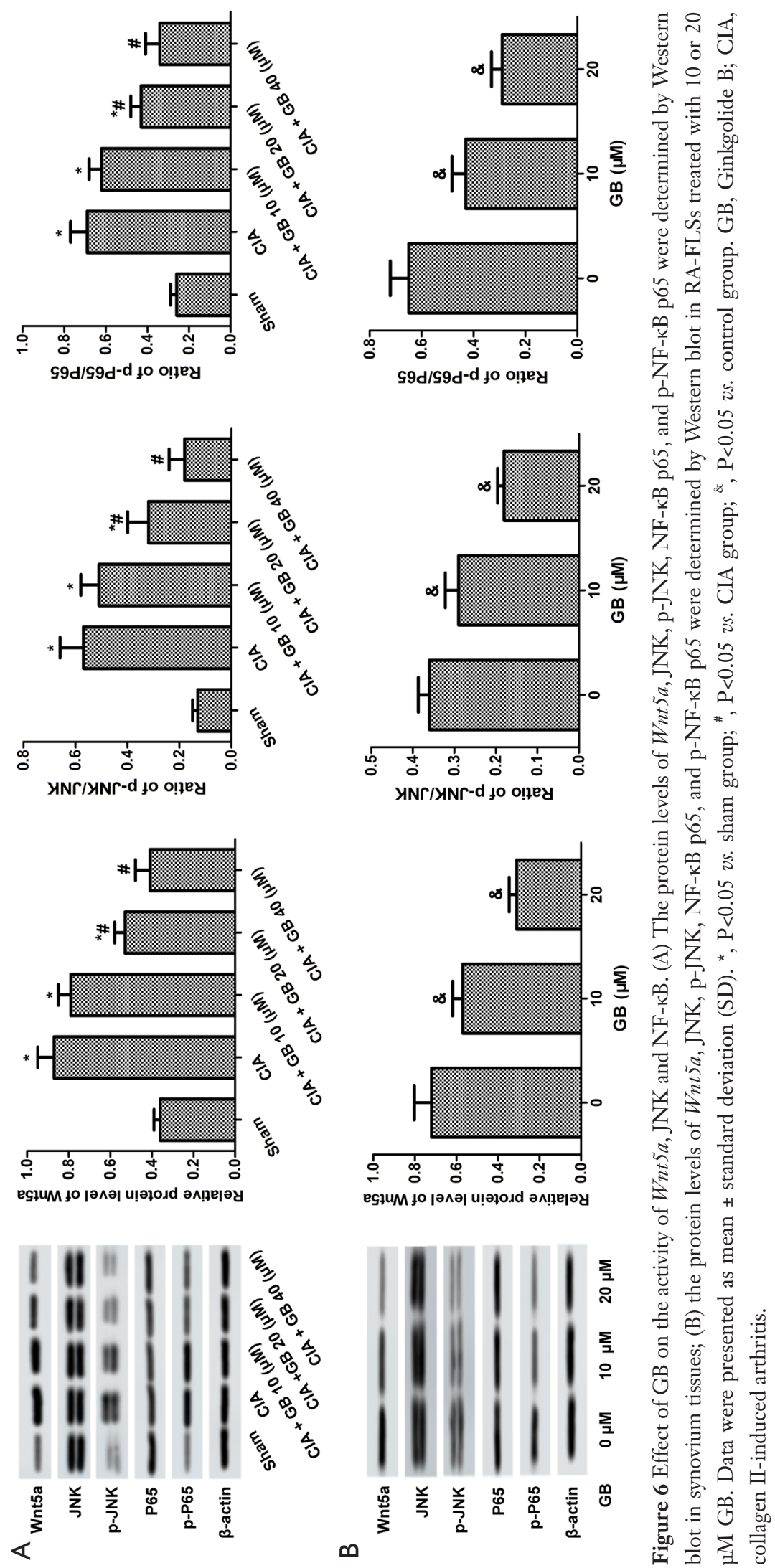

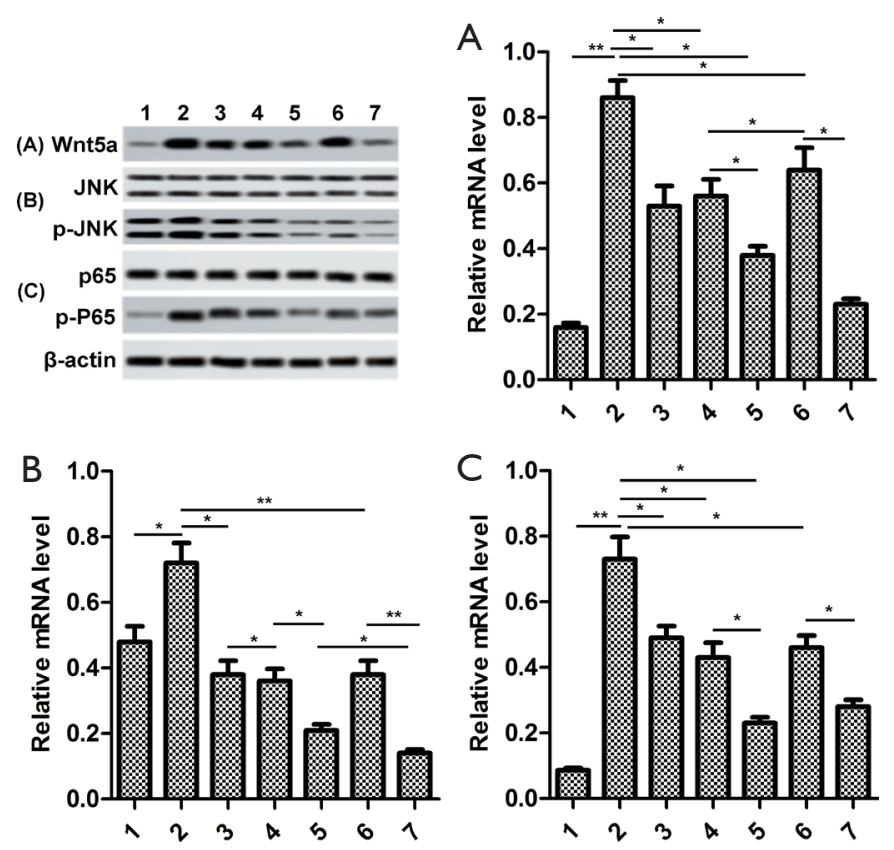

Figure 7 GB suppresses Wnt5a/JNK/NF- $\kappa$ B signaling in RA-FLSs. (A,B,C) Post adding si-Wnt5a or si-Wnt5a in RA-FLSs, the protein

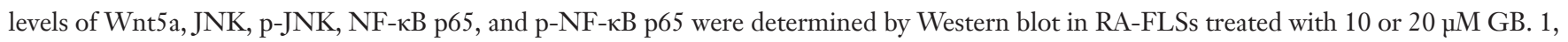
FLSs group; 2, RA-FLSs group; 3, GB group; 4, si-Wnt5a group; 5, GB+siWnt5a; 6, SP600125 group; 7, GB+SP600125 group. Data were presented as mean \pm standard deviation $(\mathrm{SD}) .{ }^{*}, \mathrm{P}<0.05 ;{ }^{* *}, \mathrm{P}<0.01$. GB, Ginkgolide $\mathrm{B}$.

synovium of arthritis (37). In this study, GB has been shown to prevent p65 phosphorylation in RA tissues and LPSinduced FLSs. Up-regulation of mitogen-activated protein kinases (MAPKs) activated the release of pro-inflammatory cytokines (TNF- $\alpha$ and IL-1 $\beta$ ) in the chronic inflammatory response of RA (38). Western blot data demonstrated that GB reduced JNK phosphorylation in vitro and in vivo, thereby inhibiting MAPK signal transduction. Nevertheless, it remains unclear whether NF- $\kappa \mathrm{B}$ and JNK signaling are direct targets for GB, and further studies are needed to elucidate the exact mechanisms by which GB regulates the NF-кB/JNK pathways. The Wnt proteins, Wnt-1 or Wnt$5 \mathrm{a}$, mediate the metabolic activity of synovial fibroblasts, such as the expression of IL- 6 and IL-15, which promotes the survival of these synovial fibroblasts (39). As an inflammatory response mediator derived from macrophages, $W n t 5 a$ accelerates the release of other inflammatory factors through autocrine or paracrine signaling (40). Furthermore, $W n t 5 a$ activates downstream NF- $\mathrm{kB} / \mathrm{JNK}$ signals, crosstalk with other signaling pathways, and participates in cellular inflammatory responses (41). The results of this study showed that GB was protective against the joint injury and inflammation caused by RA through the $W n t 5 a / J N K / N F-$ $\kappa \mathrm{B}$ pathway.

\section{Conclusions}

In summary, GB had anti-inflammatory and joint protective effects in CIA mice models. Moreover, GB induced apoptosis and inhibited the release of pro-inflammatory factors in LPS induced FLSs. These results suggested that GB alleviated arthritis and bone loss through $W n t 5 a / \mathrm{JNK} /$ $\mathrm{NF}-\kappa \mathrm{B}$ pathways. However, the potential mechanism of these results requires further investigation. Our current study confirmed the anti-RA characteristics of GB and provided new ideas for the treatment of RA.

\section{Acknowledgments}

Funding: None.

\section{Footnote}

Reporting Checklist: The authors have completed the ARRIVE reporting checklist. Available at http://dx.doi. org/10.21037/atm-20-6420 
Data Sharing Statement: Available at http://dx.doi. org/10.21037/atm-20-6420

Conflicts of Interest: All authors have completed the ICMJE uniform disclosure form (available at http://dx.doi. org/10.21037/atm-20-6420). The authors have no conflicts of interest to declare.

Ethical Statement: The authors are accountable for all aspects of the work in ensuring that questions related to the accuracy or integrity of any part of the work are appropriately investigated and resolved. Animal protocols in this study were approved by the Animal Care and Use Committee of North Sichuan Medical College. All animal experiments were performed in accordance with the guidelines of the Animal Care and Use Committee of North Sichuan Medical College.

Open Access Statement: This is an Open Access article distributed in accordance with the Creative Commons Attribution-NonCommercial-NoDerivs 4.0 International License (CC BY-NC-ND 4.0), which permits the noncommercial replication and distribution of the article with the strict proviso that no changes or edits are made and the original work is properly cited (including links to both the formal publication through the relevant DOI and the license). See: https://creativecommons.org/licenses/by-nc-nd/4.0/.

\section{References}

1. McInnes IB, Schett G. The pathogenesis of rheumatoid arthritis. New Engl J Med 2011;365:2205-19.

2. Abuwarwar MH, Knoblich K, Fletcher AL. A pathogenic hierarchy for synovial fibroblasts in rheumatoid arthritis. Ann Transl Med 2018;6:S75.

3. Sardar S, Kerr A, Vaartjes D, et al. The oncoprotein TBX3 is controlling severity in experimental arthritis. Arthritis Res Ther 2019;21:16.

4. Chin CY, Hsieh SY, Tseng VS. eDRAM: effective early disease risk assessment with matrix factorization on a large-scale medical database: a case study on rheumatoid arthritis. PLoS One 2018;13:e0207579.

5. Dulos J, Wijnands FP, van den Hurk-van Alebeek JA, et al. p38 inhibition and not MK2 inhibition enhances the secretion of chemokines from TNF-alpha activated rheumatoid arthritis fibroblast-like synoviocytes. Clin Exp Rheumatol 2013;31:515-25

6. Alsaleh G, François A, Knapp AM, et al. Synovial fibroblasts promote immunoglobulin class switching by a mechanism involving BAFF. Eur J Immunol 2011;41:2113-22.

7. Perry E, Howes MJ. Medicinal plants and dementia therapy: herbal hopes for brain aging? CNS Neurosci Ther 2011;17:683-98.

8. Yang Y, Li Y, Wang J, et al. Systematic investigation of Ginkgo Biloba leaves for treating cardio-cerebrovascular diseases in an animal model. ACS Chem. Biol 2017;12:1363-72.

9. Nash KM, Shah ZA. Current perspectives on the beneficial role of Ginkgo Biloba in neurological and cerebrovascular disorders. Integr Med Insights 2015;10:1-9.

10. Zheng PD, Mungur R, Zhou HJ, et al. Ginkgolide B promotes the proliferation and differentiation of neural stem cells following cerebral ischemia/reperfusion injury, both in vivo and in vitro. Neural Regen Res 2018;13:1204-11.

11. Mahmoud F, Abul H, Onadeko B, et al. In vitro effects of Ginkgolide B on lymphocyte activation in atopic asthma: comparison with cyclosporin A. Jpn J Pharmacol 2000;83:241-5.

12. Zhu B, Xue F, Zhang CQ, et al. Ginkgolide B promotes osteoblast differentiation via activation of canonical Wnt signalling and alleviates osteoporosis through a bone anabolic way. J Cell Mol Med 2019;23:5782-93.

13. Hu H, Li Y, Xin Z, et al. Ginkgolide B exerts antiinflammatory and chondroprotective activity in LPSinduced chondrocytes. Adv Clin Exp Med 2018;27:913-20.

14. Nusse R, Varmus H. Three decades of Wnts: a personal perspective on how a scientific field developed. EMBO J 2012;31:2670-84.

15. Lerner UH, Ohlsson C. The WNT system: background and its role in bone. J Intern Med 2015;277:630-49.

16. Kumawat K, Gosens R. WNT-5A: signaling and functions in health and disease. Cell Mol Life Sci 2016;73:567-87.

17. Shi S, Man Z, Li W, et al. Silencing of Wnt5a prevents interleukin-1beta-induced collagen type II degradation in rat chondrocytes. Exp Ther Med 2016;12:3161-6.

18. Shen HH, Yang CY, Kung CW, et al. Raloxifene inhibits adipose tissue inflammation and adipogenesis through Wnt regulation in ovariectomized rats and 3 T3-L1 cells. J Biomed Sci 2019;26:62.

19. Miyoshi M, Liu S. Collagen-induced arthritis models. Methods Mol Biol 2018;1868:3-7.

20. Yeremenko N, Harle P, Cantaert T, et al. The cartilage protein melanoma inhibitory activity contributes to infammatory arthritis. Rheumatology (Oxford) 
2014;53:438-47.

21. Camps M, Ruckle T, Ji H, et al. Blockade of PI3Kgamma suppresses joint inflammation and damage in mouse models of rheumatoid arthritis. Nat Med 2005;11:936-43.

22. Wu J, Fan KJ, Wang QS, et al. DMY protects the knee joints of rats with collagen-induced arthritis by inhibition of NF- $\kappa \mathrm{B}$ signaling and osteoclastic bone resorption. Food Funct 2020;11:6251-64.

23. Liu XZ, Fan J, Qi K, et al. Dishevelled2 promotes apoptosis and inhibits inflammatory cytokine secretion in rheumatoid arthritis fibroblast-like synoviocytes through crosstalk with the NF-kappaB pathway. Oncotarget 2017;8:12649-63.

24. Chen X, Shen J, Zhao JM, et al. Cedrol attenuates collagen- induced arthritis in mice and modulates the inflammatory response in LPS-mediated fibroblast-like synoviocytes. Food Funct 2020;11:4752-64.

25. Bartok B, Firestein GS. Fibroblast-like synoviocytes: key effector cells in rheumatoid arthritis. Immunol Rev 2010;233:233-55.

26. McInnes IB, Schett G. Cytokines in the pathogenesis of rheumatoid arthritis. Nat Rev Immunol 2007;7:429-42.

27. Kalden JR, Schulze-Koops H. Immunogenicity and loss of response to TNF inhibitors: implications for rheumatoid arthritis treatment. Nat Rev Rheumatol 2017;13:707-18.

28. Tang Y, Huang B, Sun L, et al. Ginkgolide B promotes proliferation and functional activities of bone marrowderived endothelial progenitor cells: involvement of Akt/ eNOS and MAPK/p38 signaling pathways. Eur Cell Mater 2011;21:459-69.

29. Siebert S, Tsoukas A, Robertson J, et al. Cytokines as therapeutic targets in rheumatoid arthritis and other inflammatory diseases. Pharmacol Rev 2015;67:280-309.

30. Fan Z, Yang B, Ross RP, et al. Protective effects of Bifidobacterium adolescentis on collagen-induced arthritis in rats depends on timing of administration. Food Funct 2020;11:4499-511.

31. Dayer JM. The pivotal role of interleukin-1 in the clinical manifestations of rheumatoid arthritis. Rheumatology

Cite this article as: Xie C, Jiang J, Liu J, Yuan G, Zhao Z. Ginkgolide B attenuates collagen-induced rheumatoid arthritis and regulates fibroblast-like synoviocytes-mediated apoptosis and inflammation. Ann Transl Med 2020;8(22):1497. doi: 10.21037/atm-20-6420
(Oxf) 2003;42:3ii-10.

32. Zhai KF, Duan H, Luo L, et al. Protective effects of paeonol on inflammatory response in IL-1beta-induced human fibroblast-like synoviocytes and rheumatoid arthritis progression via modulating NF-kappaB pathway. Inflammopharmacology 2017;25:523-32.

33. Choi JK, Oh HM, Park JH, et al. Salvia plebeia extract inhibits the inflammatory response in human rheumatoid synovial fibroblasts and a murine model of arthritis. Phytomedicine 2015;22:415-22.

34. Zheng CJ, Zhao XX, Ai HW, et al. Therapeutic effectsof standardized Vitex negundo seeds extract on complete Freund's adjuvant induced arthritis in rats. Phytomedicine 2014;21:838-46.

35. Burrage PS, Mix KS, Brinckerhoff CE. Matrix metallo proteinases: Role in arthritis. Front Biosci 2006;11:529-43.

36. Murphy EP, Crean D. Molecular interactions between $\mathrm{NR} 4 \mathrm{~A}$ orphan nuclear receptors and $\mathrm{NF}-\kappa \mathrm{B}$ are required for appropriate inflammatory responses and immune cell homeostasis. Biomolecules 2015:5;1302-18.

37. Xiao Y, Liang L, Huang M, et al. Bromodomain and extraterminal domain bromodomain inhibition prevents synovial inflammation via blocking I $\mathrm{B}$ kinasedependent NF- $\kappa \mathrm{B}$ activation in rheumatoid fibroblast-like synoviocytes. Rheumatology 2016;55:173-84.

38. Paunovic V, Harnett MM. Mitogen-activated protein kinases as therapeutic targets for rheumatoid arthritis. Drugs 2013;73:101-15.

39. Nakamura Y, Nawata M, Wakitani S. Expression profiles and functional analyses of Wnt-related genes in human joint disorders. Am J Pathol 2005;167:97-105.

40. Kim J, Kim J, Kim DW, et al. Wnt5a induces endothelial inflammation via $\beta$-catenin-independent signaling. J Immunol 2010;185:1274-82.

41. Naskar D, Maiti G, Chakraborty A, et al. Wnt5a-Rac1NF-kappaB homeostatic circuitry sustains innate immune functions in macrophages. J Immunol 2014;192:4386-97.

(English Language Editor: J. Jones) 